\title{
A comparison between different wall pressure measurement devices for the separation and analysis of TBL and acoustic contributions
}

\author{
Quentin Leclere, Alice Dinsenmeyer, Edouard Salze, and Jerome Antoni
}

This is a preprint of the following chapter: Leclere et al. , A comparison between different wall pressure measurement devices for the separation and analysis of TBL and acoustic contributions, published in Flinovia-Flow Induced Noise and Vibration Issues and Aspects-III, edited by Ciappi, E., De Rosa, S., Franco, F., Hambric, S.A., Leung, R.C.K., Clair, V., Maxit, L., Totaro, N., 2021, Springer, reproduced with permission of Springer (as it appears on the copyright page of the book). The final authenticated version is available online at: https://www.springer.com/gp/book/9783030648060.

\begin{abstract}
The wall pressure measured by an array of flush mounted microphones subject to a flow results from two components. The first one is due to the pressure fluctuations generated by vortices convected by the flow in the turbulent boundary layer developed on the wall, and the second one results from acoustic waves that are emitted by some acoustic sources. Different type of sensor arrays can be used to characterize the first or second components, or even both. In all cases, a major difficulty is to separate as well as possible their contributions to the measurements. Different techniques are reviewed in this chapter to achieve this goal, based on either the measurement device itself or the post processing method. All approaches are implemented in a unique experimental setup, conducted in the frame of the ADAPT Clean Sky 2 project, allowing to objectively highlight their advantages and drawbacks.
\end{abstract}

Quentin Leclere, Alice Dinsenmeyer, and Jerome Antoni

Univ Lyon, INSA-Lyon, Laboratoire Vibrations Acoustique, F-69621 Villeurbanne, France. e-mail: quentin.leclere@insa-lyon.fr

Edouard Salze

Univ Lyon, Ecole Centrale de Lyon, Laboratoire de Mecanique des Fluides et d'Acoustique, F69134, Ecully, France. 


\section{Introduction}

Microphone array measurements are commonly used for the characterization of aeroacoustic sources in wind tunnels. The object of interest (or a mockup of it) is installed in the tunnel and a flow is established so as to generate aeroacoustic sources. The difficulty, in these conditions, is that the pressure fluctuations observed at one given point in the tunnel result from the acoustic pressure, radiated by the aeroacoustic source of interest, but also from fluctuations caused by the flow itself. For practical reasons, microphones are often installed on a wall of the tunnel. The acoustic pressure radiated by the source of interest is then disturbed by the pressure due to the turbulent boundary layer developed on the wall, with often strongly negative signal to noise ratios (SNRs). The post-processing of such data, for acoustic imaging purpose, requires the separation of these two contributions (acoustic and TBL). This separation relies on statistical and/or physical properties of these two components.

Statistically, correlation lengths of the TBL are very short, the resulting coherence between different sensors of the array being very low. The contribution of the TBL to averaged cross-spectral quantities is thus limited (except for auto-spectra). Many acoustic imaging techniques are based on the post-processing of averaged crossspectral quantities, either excluding [1] or reconstructing [2] auto-spectra. These approaches require averaged cross-spectra, which in turn requires quite long time records. Moreover, transient acoustic events cannot be analyzed using such averaged spectral quantities.

Other separation techniques rely on the different physical properties of the TBL and acoustic fields, more specifically their properties in the wavenumber domain. The acoustic part is by definition limited to the low wavenumber domain (lower or equal to the acoustic wavenumber). The support of the TBL is much wider, with a maximum around the convective wavenumber in the direction of the flow. A separation of acoustic and TBL contributions can thus be processed by a filtering operation in the wavenumber domain. This filtering can be operated either by post-processing wall pressure fields sampled using high density microphone arrays, or alternatively using a low-pass physical filtering operating before data acquisition. Post-processing filtering requires high density microphone arrays to avoid aliasing problems at the stage of spatial sampling, the high number of required microphones is often reached using multi-pass measurements [3]. Physical filtering is achieved by various ways, using surface or remote microphones, the filtering being realized by an averaging of the pressure over a surface determined by the sensor area or by the section of the tube connected to remote sensors [3, 4]. Another possibility is to use filtering properties of thin structures: a thin plate submitted to a wall pressure field will act as a low pass filter, with a cut-off frequency equal to its natural wavenumber. The vibration of the plate can be used to recover the low wavenumber part of the incident pressure field using inverse methods [5, 6].

The aim of the present contribution is to compare different experimental approaches to separate TBL and acoustic contributions in the frame of a unique academic experiment. The experimental setup has been realized in the frame of the ADAPT Cleansky 
EU project, in a small wind tunnel at Ecole Centrale de Lyon. The first section of this work is dedicated to the experimental setup, including a detailed description of the three different sensor arrays that have been tested. The beamforming processing that is used to analyze the measured pressure fields is presented in a second section, with an original way of formulating the quadratic problem using vectorization, offering interesting options to easily process partial CSMs (Cross Spectral Matrices). The third section finally enumerates different separation techniques based on physical assumptions, statistical properties, or spatial filtering approaches with their basic principle and hypothesis, together with some illustrations based on the different datasets.

In order to ease the reading of the work, a list of main acronyms used in the text is provided hereafter.

- ADAPT: ADvanced Aeroacoustic Processing Techniques (EU project)

- CSM: Cross Spectral Matrix

- CS(s): Cross Spectrum (Spectra)

- MEMS: Micro Eletro-Mechanical System

- PCB: Printed Circuit Board

- PDF: Probability Density Function

- PFA: Probabilistic Factor Analysis

- SNR: Signal to Noise Ratio

- TBL: Turbulent Boundary Layer

\section{Experimental setup}

The experimental campaign described in this section has been conducted in the frame of the ADAPT Cleansky EU project. The experiment was conducted in the main subsonic wind tunnel at Ecole Centrale de Lyon, LMFA, Centre Acoustique. The flow was generated using a $850 \mathrm{~kW}$ Houden centrifugal blower. Air passes through settling chambers including honeycombs and several wire meshes designed to reduce freestream turbulence. For the project, a closed-section wind tunnel was used (see Fig. 1). The first section of the wind tunnel is a 2-meter long zero-gradient tunnel, designed to measure wall-pressure fluctuations beneath a turbulent boundary layer. The second section is a divergent tunnel, designed to lower the exhaust flow speed. The side walls and roof of these two sections are equipped with porous liners, so as to limit acoustic reflections. The third section is an exponential horn, also with an acoustic treatment, designed to suppress the acoustical reflections on the exhaust of the tunnel.

Three acoustic sources are mounted in the roof of the tunnel. Two of them are placed very close to one another, and wired in opposite phase, so as to generate a dipole type source. The third source is placed at about $20 \mathrm{~cm}$ from the two former, and is driven by an independent generator so as to obtain incoherent sources. 


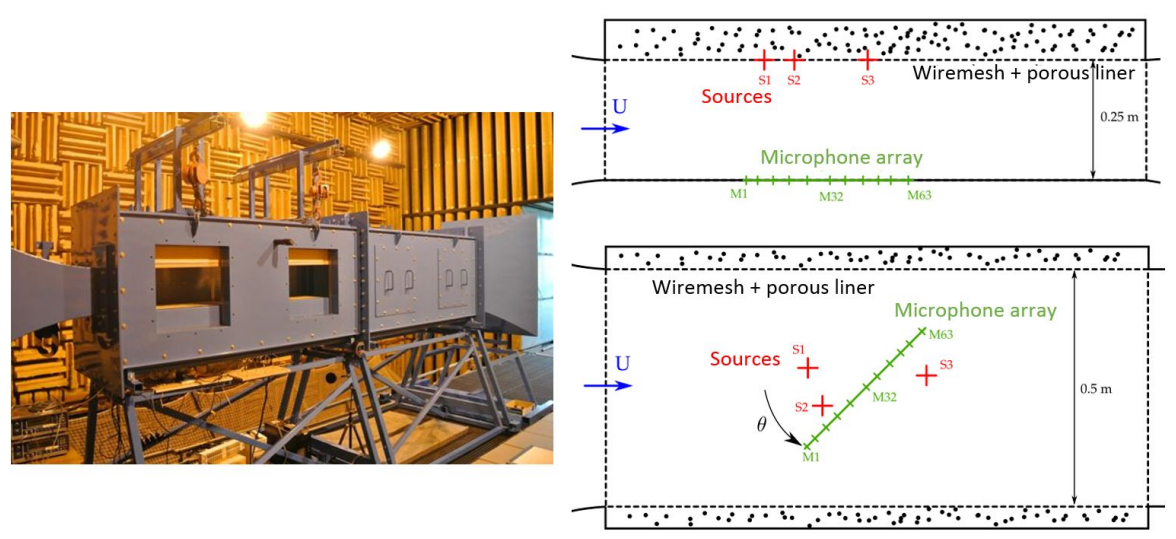

Fig. 1 Left: Picture of the wind tunnel. Right : sketch of the test section, side view (top) and top view (bottom).
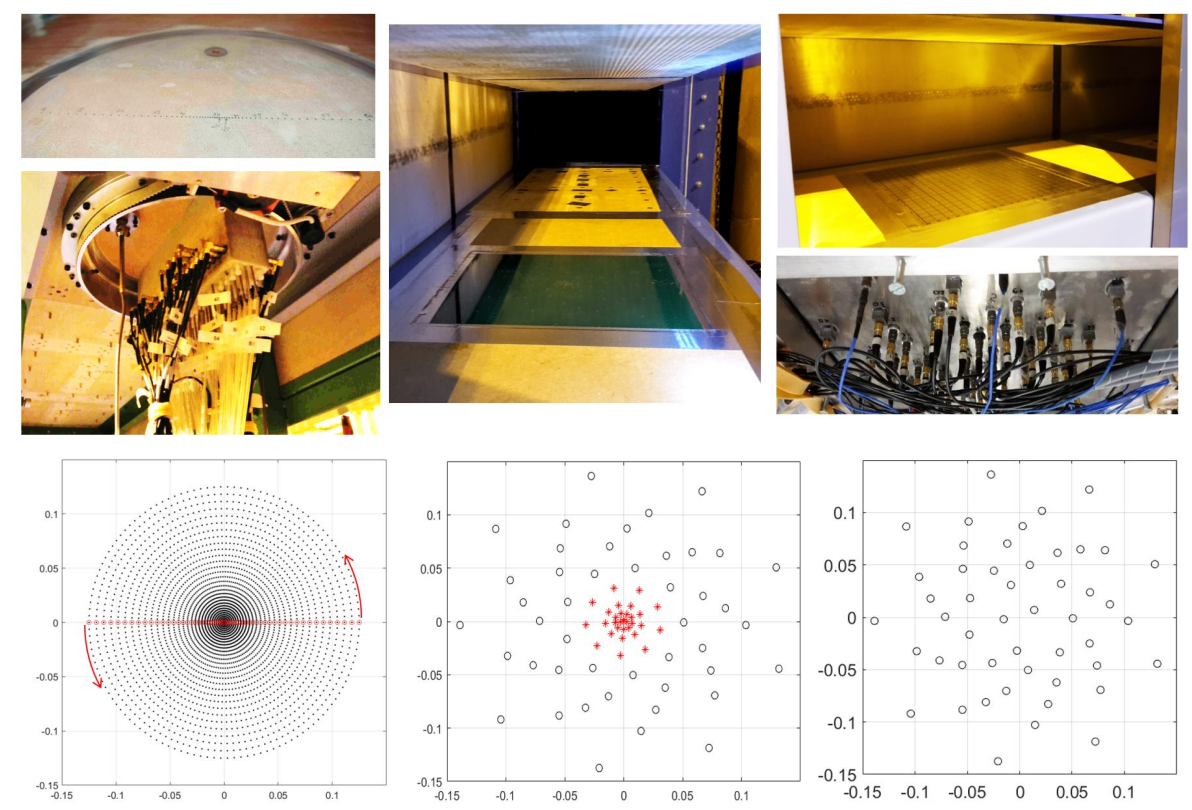

Fig. 2 Top, from left to right : the rotating linear array, the MEMS array, the accelerometer array. Bottom : corresponding sensor layouts.

Three different wall pressure field measurement devices are successively mounted in the floor of the test section (see Fig. 2):

- The rotating linear array. 63 remote microphone probes are mounted on a rotating disk, following a linear non uniform distribution (see Fig. 2). The smallest distance between two neighbour microphones is $1 \mathrm{~mm}$, and the largest distance 
is $250 \mathrm{~mm}$. A number of 63 angular positions has been retained for this study, from 0 to 180 degrees. For each measurement configuration, and each angular position of the array, the 63 signals are recorded simultaneously during 60s (sampling frequency $51.2 \mathrm{kHz}$ ), which makes a total of 3907 measurement positions. However, the full cross spectral matrix is not measured : a cross spectral matrix is computed for each array position, cross spectra are thus measured only between measurement positions belonging to the same acquisition (same array position). It means that considering the whole CSM between the 3907 measured positions, only about $250 \times 10^{3}$ cross spectra are measured, over the $3907^{2} \approx 15 \times 10^{6}$ elements of the full CSM (which means that about $1.5 \%$ of the full CSM is actually measured). However, it represents a quite heavy amount of data, that necessitates also a long measurement time $(60 s \times 63$ positions, only considering acquisition duration) for each measurement configuration.

- The MEMS array. 76 digital MEMS microphones are distributed on the backside of a PCB (Printed Circuit Board), with pinholes in front of each sensor. The microphone arrangement has a spiral-like distribution of 9 arms for 45 sensors, classically used for acoustic imaging purposes (see Fig. 2, black o markers). The last 41 microphones (red star markers) are distributed in the central part of the PCB using a denser polar-like distribution, so as to obtain more information about high wavenumbers. Four additional channels are used for 2 accelerometers glued on the backside of the PCB and 2 microphones placed in a small cavity facing the backside of the PCB (the frontside being exposed to the flow). These additional channels are expected to be much less affected by the flow than wall pressure sensors, because of the filtering effect the PCB plate - intending to use them as references to apply referenced-based denoising techniques. All channels are acquired simultaneously, at a sampling frequency of $51.2 \mathrm{kHz}$, a full $80 \times 80 \mathrm{CSM}$ is measured for each configuration.

- The accelerometer array. 49 accelerometers (weight $5 \mathrm{mg}$ each, bandwidth [0.1$10] \mathrm{kHz}$ ) are fixed on a thin aluminium plate (thickness $1 \mathrm{~mm}$ ), with a layout similar to the MEMS array, at least for the 45 spiral subset. Four additional sensors are fixed in the central part (instead of 31 for the MEMS microphone array). The wavenumber filtering effect of the plate implies that high wavenumbers will not excite the plate, it is thus not necessary to have a high density distribution. The excitation pressure field has to be recovered from the plate vibration measured by the 49 accelerometers. The inverse method that is necessary to achieve this goal is described in section 4.4. The 49 accelerations are acquired simultaneously, during 30s (sampling frequency $64 \mathrm{kHz}$ ), so as to fill-in the whole $49 \times 49 \mathrm{CSM}$ for each measurement configuration. Note that the mass of accelerometers, as well as the cables, have a significant effect on the dynamic behaviour of the plate. This aspect is discussed in section 4.4.

For the three measurement devices, CSMs are estimated using the Welch's method, with a frequency resolution of $12 \mathrm{~Hz}$ (snapshot length of about $83 \mathrm{~ms}$, Hanning window, overlap 66\%). 

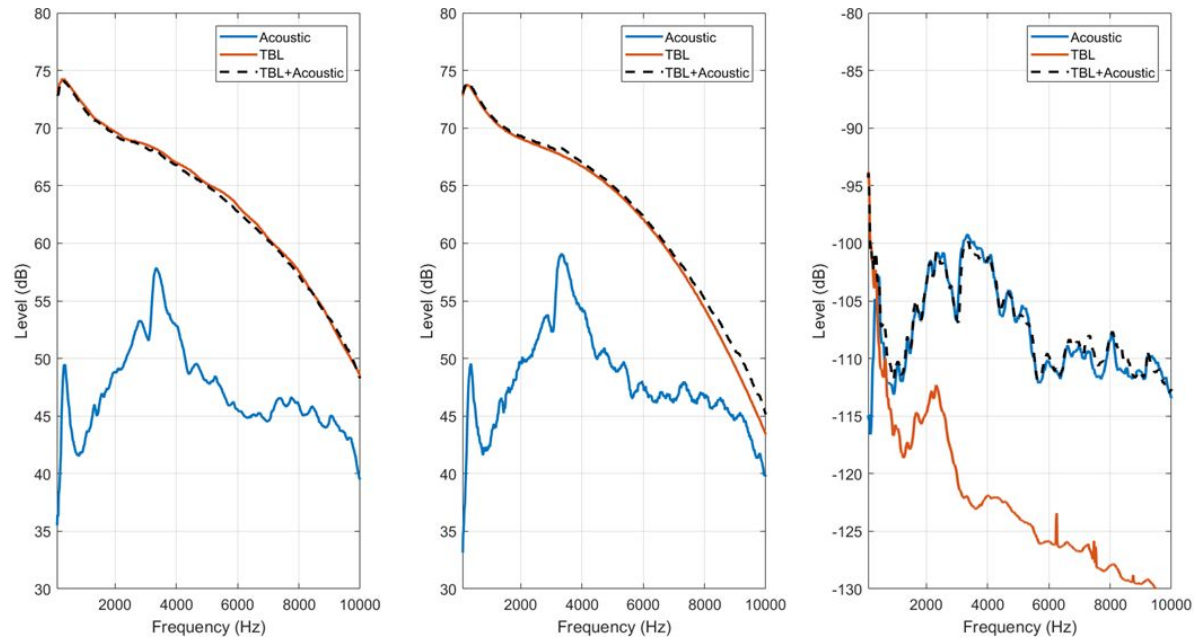

Fig. 3 Averaged autospectra measured by the different arrays for configuration A (solid blue), T30 (solid red) and AT30 (dashed black). From left to right : averaged pressure autospectra of the rotating array, averaged pressure autospectra of the MEMS array, averaged acceleration autospectra of the accelerometer array.

Different measurement configurations are considered, with or without acoustic sources, with or without flow, for different flow speeds. Results presented in this work focus on the 3 following configurations :

- Configuration A: no flow, acoustic sources switched on.

- Configuration T30: flow speed at $30 \mathrm{~m} / \mathrm{s}$, acoustic sources switched off.

- Configuration AT30: flow speed at $30 \mathrm{~m} / \mathrm{s}$, acoustic sources switched on.

As a preliminary result, the average autospectra obtained for each array and each configuration are displayed in Fig. 3. Averaged pressure autospectra obtained by the two microphone arrays are very similar. The contribution of the TBL is much stronger on the whole frequency range, the acoustic to TBL ratio lying between $-25 \mathrm{~dB}$ at low frequencies and $-5 \mathrm{~dB}$ at high frequencies. The averaged accelerometer autospectrum is, as expected, different from pressure autospectra (measured accelerations resulting from the response of the plate to the pressure field). However, it is interesting to note that for the accelerometer array, the acoustic to TBL ratio is much favourable than for the two former, lying between $0 \mathrm{~dB}$ at low frequency and +15 to $+20 \mathrm{~dB}$ at high frequency. This preliminary result illustrates well the interest of using accelerometers instead of microphones if one is interested in the acoustic part : the gain in terms of SNR can be estimated roughly to $25 \mathrm{~dB}$ in this case. 


\section{Beamforming processing}

Beamforming is a well known processing method for microphone array measurements. Let $\mathbf{p}$ be the complex pressure field measured at a given frequency on the microphone array, and $\mathbf{w}_{\mathbf{i}}$ the theoretical complex contribution of a given wave (plane or spherical), indexed $i$, at microphone positions. The beamforming output associated to this particular wave is noted $\left|q_{i}\right|^{2}$, where the complex coefficient $q_{i}$ minimizes the quantity $\left\|\mathbf{p}-q_{i} \mathbf{w}_{\mathbf{i}}\right\|$. It is the solution of a classical linear regression problem, corresponding to the projection of $\mathbf{p}$ on $\mathbf{w}$; it is equal to

$$
q_{i}=\frac{\mathbf{w}_{\mathbf{i}}{ }^{H} \mathbf{p}}{\|\mathbf{w}\|^{2}},
$$

where subscript ${ }^{H}$ stands for the hermitian transpose. $\left|q_{i}\right|^{2}$ is then represented as a function of wave parameters, either wavenumber (plane wave beamforming) or source position (spherical wave beamforming). In aeroacoustics, considering the random nature of source phenomena, the acoustic pressure field is generally available under the form of an averaged CSM, representing experimental estimations of expected values of auto and cross spectra of microphone signals. That is why Eq. (1) is commonly written in a quadratic form :

$$
\left|q_{i}\right|^{2}=\frac{\mathbf{w}_{\mathbf{i}}^{H} \mathbf{S}_{\mathbf{p p}} \mathbf{w}_{\mathbf{i}}}{\left\|\mathbf{w}_{\mathbf{i}}\right\|^{4}}
$$

Still considering aeroacoustic applications, it is generally recommended to set to zero the diagonal of the CSM to remove the contribution of incoherent noise. The right part of Eq. (2) is in this case not always positive anymore, the resulting squared source amplitude can be set to zero when found negative. In the literature, Eq. (2) is sometimes formulated in a vectorized form,

$$
S_{i i}=\frac{\operatorname{Vec}\left(\mathbf{w}_{\mathbf{i}} \mathbf{w}_{\mathbf{i}}{ }^{H}\right)^{H} \operatorname{Vec}\left(\mathbf{S}_{\mathbf{p p}}\right)}{\left\|\operatorname{Vec}\left(\mathbf{w}_{\mathbf{i}} \mathbf{w}_{\mathbf{i}}{ }^{H}\right)\right\|^{2}},
$$

where $S_{i i}$ is an estimation of the expected value of $\left|q_{i}\right|^{2}$ and where Vec (A) stands for a vector resulting from the vertical concatenation of columns of matrix A. Eq. (3) is strictly equivalent to (2), however it is interesting to note also the similarity with Eq. (1), where it appears explicitely that $S_{i i}$ is the coefficient of the linear regression operated on quadratic values (minimization of $\left.\left\|\operatorname{Vec}\left(\mathbf{S}_{\mathbf{p p}}\right)-S_{i i} \operatorname{Vec}\left(\mathbf{w}_{\mathbf{i}} \mathbf{w}_{\mathbf{i}}{ }^{H}\right)\right\|\right)$.

An interesting option considering the vectorized form is that it is possible to skip some terms in the vectorization operation of both $\mathbf{S}_{\mathbf{p p}}$ and $\mathbf{w}_{\mathbf{i}} \mathbf{w}_{\mathbf{i}}{ }^{H}$, for instance autospectra. This leads to a result similar to the diagonal removal operation, yet with a slightly different normalisation term. It is also advised, in order to optimize the computational cost, to skip the whole upper or lower part of vectorized matrices, which are hermitian (the information being redundant). By doing so, only the real part of the resulting value of $S_{i i}$ should be considered. In some situation, the whole 

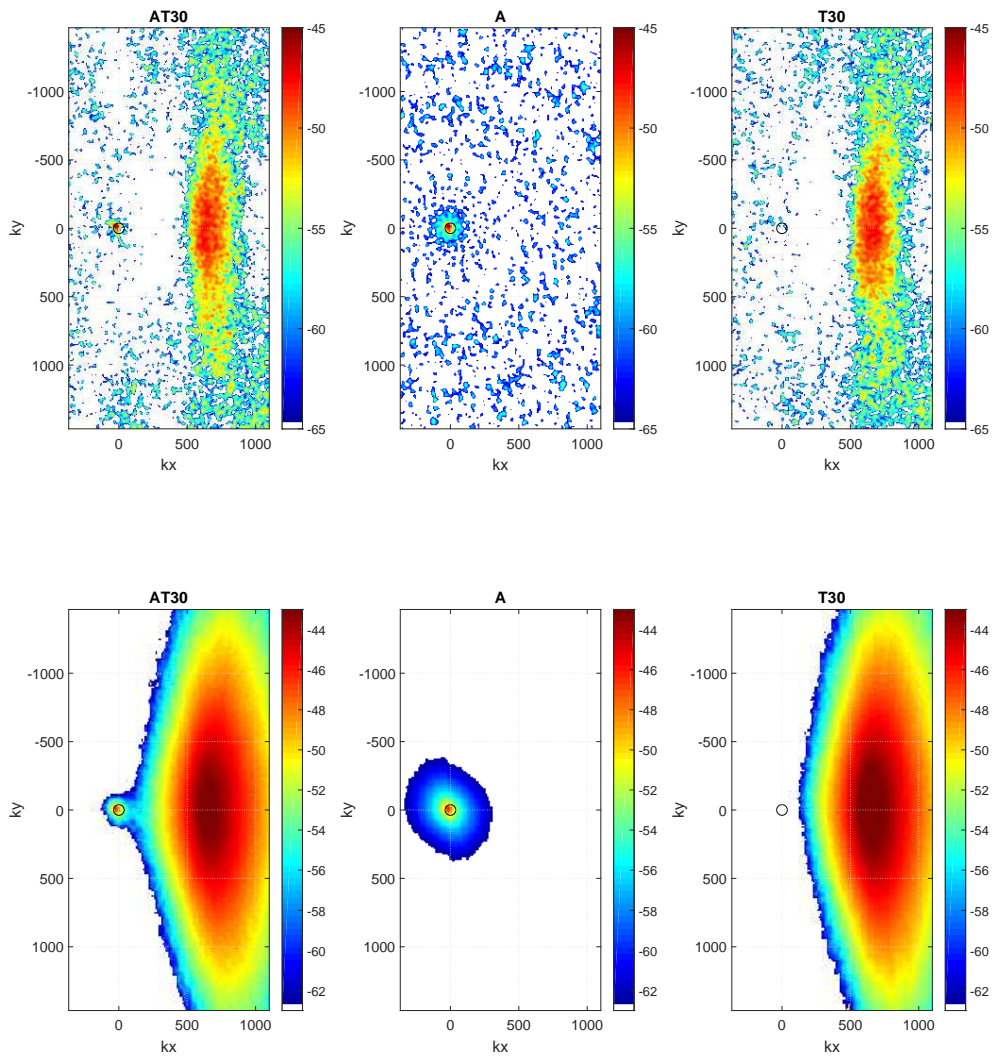

Fig. 4 Plane wave beamforming maps obtained for measurement configurations AT30, A, T30 (left to right) using the MEMS array (top) and the rotating array (bottom) at $2100 \mathrm{~Hz}$.

CSM $\mathbf{S}_{\mathbf{p p}}$ is not available (typically if the measured data result from several array positions recorded successively). In such a case the vectorized form is easily considered only for cross spectral quantities that are actually measured. More generally, it is possible to process only a subset of measured cross spectra, following additional criteria based on geometrical of statistical consideration (this possibility is illustrated in section 4.1).

Some beamforming maps, calculated from measurements described in section 2, are drawn in Fig. 4 for a frequency equal to $2100 \mathrm{~Hz}$. Wave functions $\mathbf{w}_{\mathbf{i}}$ are here plane waves determined by their $x$ and $y$ wavenumbers $k_{x i}$ and $k_{y i}$ :

$$
\mathbf{w}_{\mathbf{i}}=\exp \left(\imath k_{x i} x\right) \exp \left(\imath k_{y i} y\right) .
$$



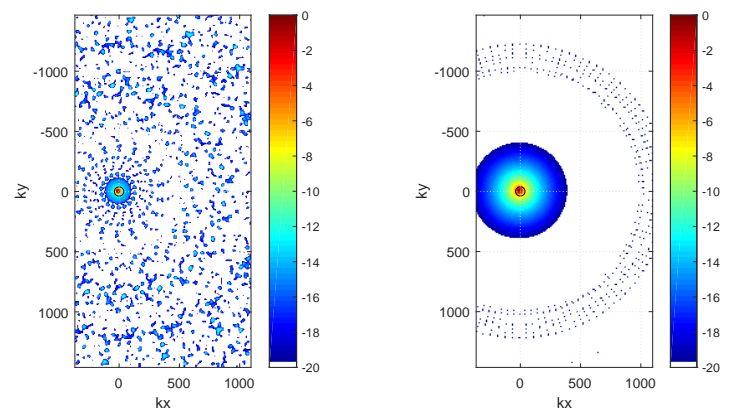

Fig. 5 Point Spread Functions of the rotating (left) and MEMS (right) arrays $(2100 \mathrm{~Hz})$, for a plane wave with normal incidence $\left(k_{x}=k_{y}=0\right)$

Beamforming is calculated using Eq. (3) from averaged CSMs (see section 2 for details), skipping autospectrum entries in the vectorization operation. Results are presented as a function of $k_{x i}$ and $k_{y i}$, for two different microphone arrays (the MEMS array and the rotating array), and for three measurement configurations with acoustic sources and TBL at 30m/s (AT30), with acoustic sources only (A), and with TBL only (T30).

Note that for the rotating array, a large proportion of the full CSM is not available because the cross spectra are measured only between measurement points that are acquired simultaneously. Missing entries are however easily skipped using the vectorized form of quadratic beamforming.

Beamforming maps allow the qualitative assessment of the wavenumber content of the measurements. For configuration A, the energy is mainly concentrated inside the acoustic circle $\left(k_{x}^{2}+k_{y}^{2}<k_{a}^{2}\right.$, where $k_{a}=\omega / c_{0}$ is the acoustic wavenumber, ratio between the angular frequency and the sound speed). With the TBL (configuration T30), the convective ridge clearly appears around $k_{x}=k_{c}$ (where $k_{c}=\omega / U_{c}$ is the convection wavenumber, ratio between the angular frequency and the convective velocity of the flow), and both contributions are observed on the map obtained from configuration AT30. An important point, when comparing results obtained with the two arrays, is that beamforming results strongly depend on the microphone array used for the measurements. Even if main features and levels are similar for the two arrays, it appears for instance that maps obtained from the rotating array are much smoother than maps obtained with the MEMS array. An explanation is simply that the rotating array is much denser, with many more measurement points. Even if a large part of its CSM is not measured, the number of effectively measured cross spectra $\left(63 \times 62 \times 63 / 2=123 \times 10^{6}\right)$ for the rotating array is much larger than the number of cross spectra measured with the MEMS array $(76 \times 75 / 2=2850)$.

Point Spread functions (PSF) of both arrays are drawn at the same frequency $(2100 \mathrm{~Hz})$ in Fig. 5, for a plane wave of normal incidence. Clearly, the PSF of the MEMS array is polluted by a lot of grating lobes caused by the lack of information. It is also seen that the main lobe is larger for the rotating array than for the MEMS 


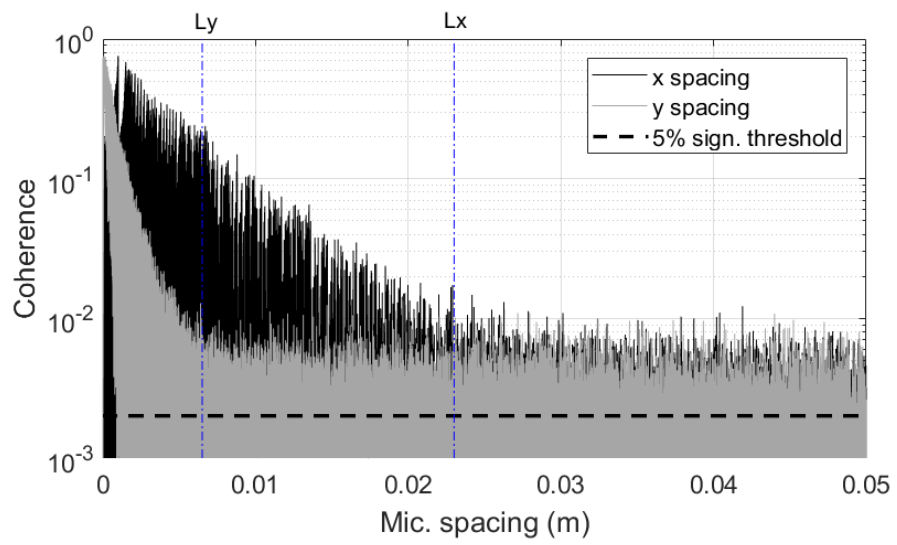

Fig. 6 Coherence between the microphone pairs of the rotating array as a function of the microphone spacing along the $\mathrm{x}$ and $\mathrm{y}$ direction.

array because of the strong heterogeneity of the rotating array microphone density that is much higher at the center of the array.

\section{Separation approaches}

\subsection{Space domain cross-spectra selection}

A first separation can be simply operated by splitting cross-spectral data into two subsets based upon geometrical considerations. It is indeed known that the turbulence can have relatively short correlation lengths, and may contribute strongly only to cross spectra of close microphone pairs. This is clearly the case for TBL analytical models like Corcos [8], that predicts an exponential decrease of the coherence as a function of the microphone pair distance. On the other hand, the contribution of an acoustic field is modulated in amplitude only by the source-microphone distance, that may not vary so much as a function of the microphone positions. The coherence of an acoustic field is thus be not too affected by the microphone distance. In order do illustrate these considerations, the coherence between microphone pairs are drawn in Fig. 6 as a function of the $x$ and $y$ spacings, for the configuration AT30 (acoustics and TBL at $30 \mathrm{~m} / \mathrm{s}$ ) for the rotating array. When the distance is close to zero, the exponential decrease predicted by the TBL analytical model is well observed, with a stronger decay rate in the $y$ direction (perpendicular to the flow). Above a given critical length, different on $x$ and $y$, the coherence reaches a floor level being not dependent on the distance anymore. It can be assumed that 

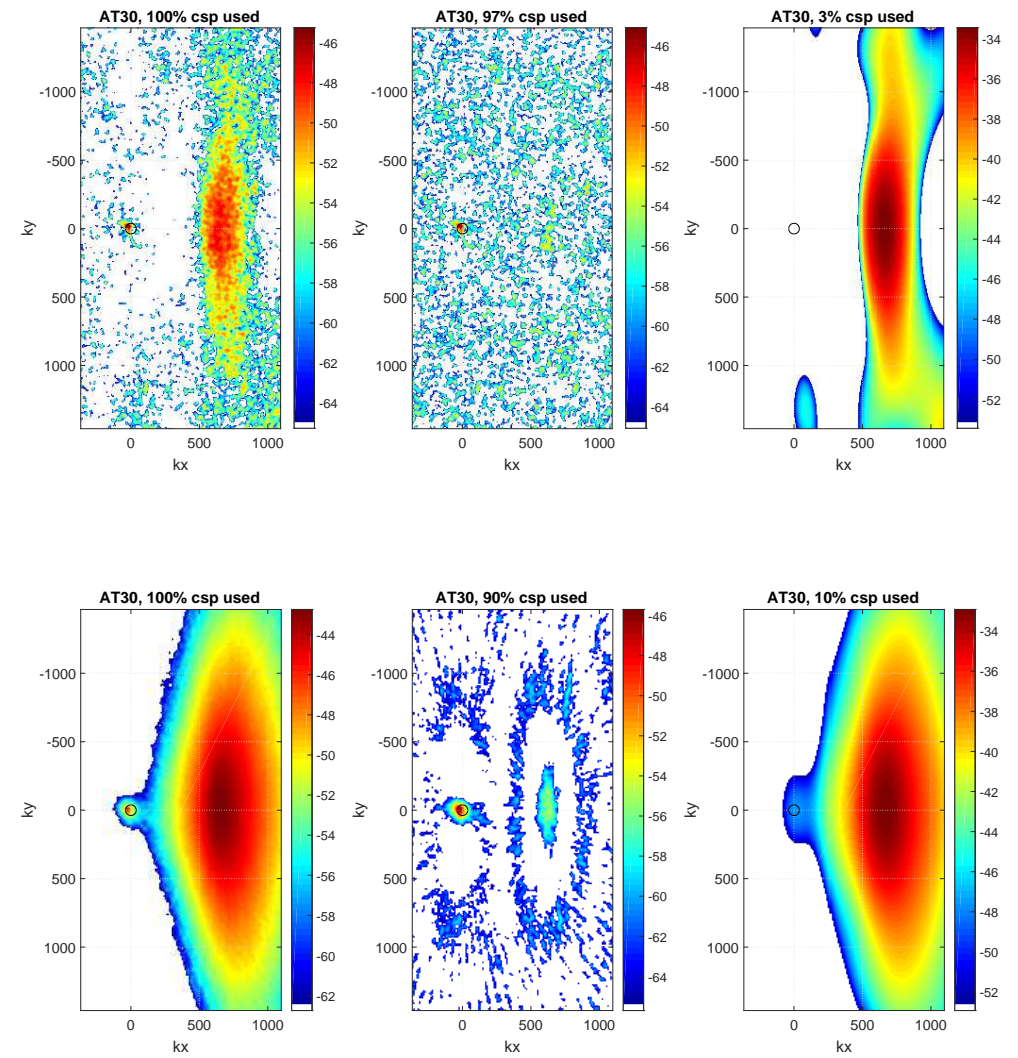

Fig. 7 Plane Wave beamforming maps obtained for the measurement configuration AT30 using the MEMS array (top) and the rotating array (bottom) at $2100 \mathrm{~Hz}$. From left to right : using all CSs, using CSs satisfying $\left(\Delta x / L_{x}\right)^{2}+\left(\Delta y / L_{y}\right)^{2}>1$, using CSs satisfying $\left(\Delta x / L_{x}\right)^{2}+\left(\Delta y / L_{y}\right)^{2}<1$.

above these critical distances noted $L_{x}$ and $L_{y}$, the acoustic field is stronger than the TBL, and inversely below. Thus, considering the vectorized quadratic formulation of beamforming, it is possible to split cross-spectral data into two subsets, a first one satisfying $\left(\Delta x / L_{x}\right)^{2}+\left(\Delta y / L_{y}\right)^{2}<1$ (resp. $\left.>1\right)$ for which the TBL (resp. acoustics) is the major contribution. Note that this criterion assumes an elliptic form of the contour of the 2D coherence function $[9,10]$.

Resulting plane wave beamforming maps at $2100 \mathrm{~Hz}$ are displayed in Fig. 7 for the rotating and MEMS arrays. They illustrate well that the information relative to the TBL is mainly carried by cross spectra of close microphones, and that distant microphones are essentially carrying acoustics. However, it is seen that the separation is not perfect : some energy of the convective ridge is still visible on beamforming maps from CSs (Cross Spectra) of distant microphones, and a bit of acoustics is 
still noticeable on the maps from CSs of close microphones (at least for the rotating array). Another difficulty related to the splitting approach is that it affects the PSFs (Point Spread Functions): maps from CS of close microphones are clearly oversmoothed, while the ones from distant microphones are more strongly polluted by secondary lobes due to the severe undersampling.

\subsection{CSM denoising}

An alternative to cross-spectra selection is to process each term of the CSM to extract the contribution of acoustics and TBL parts. In this section the physical structure of the acoustic field is not exploited, this possibility will be inspected in Section 4.3 dedicated to wavenumber filtering.

A first possibility illustrated in this section is to separate the two contributions using some reference signals, a priori known to result from one component only and in a sufficient number to represent completely this component. A second possibility is to try to proceed blindly (without any reference), but using a statistical model of the two contributions. The two approaches presented in this section are using full measured CSMs of microphones, they are thus illustrated only with the MEMS array data.

\subsubsection{Reference Based denoising}

Considering the problem of separating acoustics and TBL contributions, the reference signals cannot be used to capture the TBL, because of the statistical complexity of the field that would require an infinite number of references. On the other hand, the acoustical field is known to result from the contribution of few components, it is thus possible to represent it with few reference sensors only. The difficulty is to choose these references so as to minimize the contribution of the TBL on it. In the present application, 2 accelerometers are placed on the back side of the PCB supporting the MEMS microphones, and 2 additional microphones are positioned in the acoustic volume below the PCB. These 4 sensors are expected to benefit from the filtering effect of the PCB: in the frequency range of interest, the PCB behaves like a thin plate, filtering out high wavenumbers related to the TBL structures (this phenomenon is discussed more deeply in section 4.4). Let us denote $\mathbf{S}_{\mathbf{p p}}$ and $\mathbf{S}_{\mathbf{r r}}$ the CSMs of the microphones and the references, respectively, and $\mathbf{S}_{\mathbf{p r}}$ the matrix of cross-spectra between microphones and references. The reference microphone CSM is finally calculated by using the following equation

$$
\begin{aligned}
& \mathbf{S}_{\mathbf{p p}}{ }^{r e f}=\mathbf{S}_{\mathbf{p r}}\left(\mathbf{S}_{\mathbf{r r}}\right)^{-1} \mathbf{S}_{\mathbf{p r}}{ }^{H}, \\
& \mathbf{S}_{\mathbf{p p}}{ }^{r e s}=\mathbf{S}_{\mathbf{p p}}-\mathbf{S}_{\mathbf{p p}}{ }^{\text {ref }},
\end{aligned}
$$

where $\mathbf{S}_{\mathbf{p p}}{ }^{\text {ref }}$ is the referenced CSM, representing the part of the CSM that is coherent with the references, and where $\mathbf{S}_{\mathbf{p p}}{ }^{\text {res }}$ is the residual CSM. Note that this 

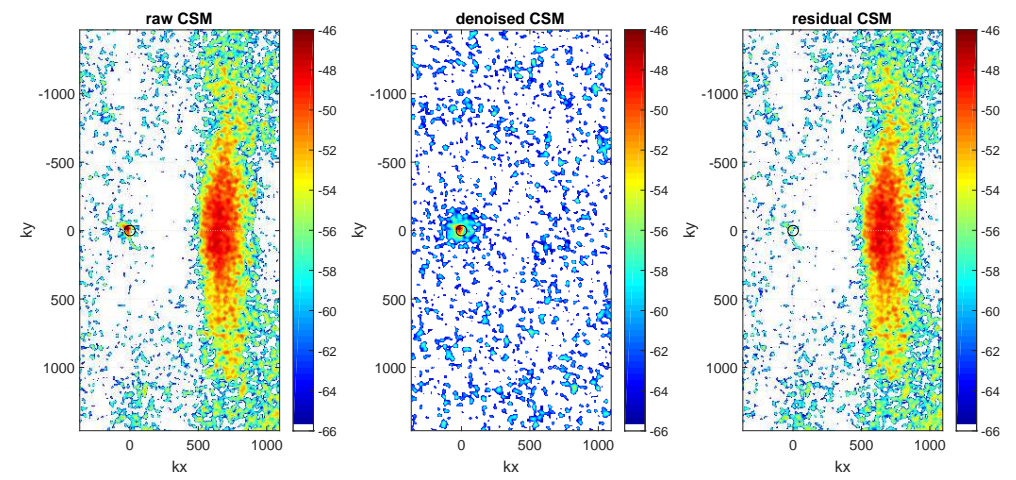

Fig. 8 Plane Wave beamforming maps obtained for the measurement configuration AT30 using the MEMS array at $2100 \mathrm{~Hz}$. From Left to right : measured CSM $\left(\mathbf{S}_{\mathbf{p p}}\right)$, reference based denoised CSM $\left(\mathbf{S}_{\mathbf{p p}}{ }^{\text {ref }}\right)$, residual CSM $\left(\mathbf{S}_{\mathbf{p p}}{ }^{\text {res }}\right)$.

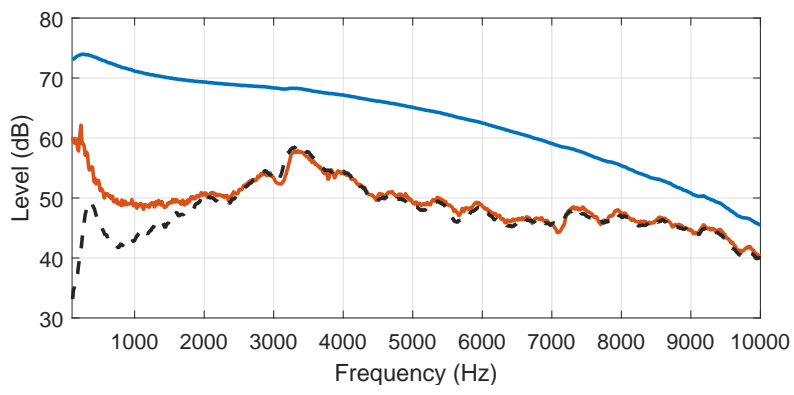

Fig. 9 MEMS array averaged Autospectra. Measurement configurations AT30 (solid blue), A (dashed black) and referenced based denoised AT30 (solid red).

approach can simply be interpreted as a generalization of the Coherent Spectrum to the multiple reference case (cf. [7]).

The efficiency of the method is illustrated on plane wave beamforming maps at $2100 \mathrm{~Hz}$, for the configuration AT30, in Fig. 8. Qualitatively, results correspond quite nicely to maps obtained from measurements T30 and A (see Fig. 4). Contrary to the results of the previous section using the basic cross-spectra selection approach, the PSF is here not affected by the separation method.

The effect of the referenced denoising approach can be also illustrated on averaged autospectra (see Fig. 9). Denoised autospectra of configuration AT30 are compared to autospectra measured with acoustics only (configuation A). Note that the contribution of acoustic sources is affected by the presence of the convection, so that perfectly 
denoised data measured with flow should not fit exactly measurements without flow. However, considering the relatively low convection velocity and short distance between microphones and sources, the effect of the presence of the flow is expected to be limited. As a matter of fact, the averaged denoised autospectrum (config. AT30) is very close to the averaged autospectrum without flow (config. A) above $2 \mathrm{kHz}$. Below $2 \mathrm{kHz}$, the referenced denoising approach performs less well, a possible reason being the very low SNR that goes below $-20 \mathrm{~dB}$. Better results may have been achieved using longer measurements to get a better convergence of statistical estimates.

\subsubsection{Blind extraction of the acoustical contribution by the use of stochastic modeling}

The Probabilistic Factor Analysis (PFA) is used in many fields to capture the underlying correlation structures in a dataset. Using the fact that the acoustical and TBL pressure fields have different correlation structures, PFA can be used to separate them. Let us assume that the measured CSM results from the sum of three major contributions, at each frequency:

1. The acoustical field produced by a small number of sources (compared to the number of sensors), with a correlation length higher than the average sensor interspacing. As explained in the previous sections, the acoustical measured spectrum can thus be statistically described by a few latent variables, at the $j^{t h}$ snapshot:

$$
\mathbf{a}_{j}=\mathbf{L} \mathbf{c}_{j}, \quad j=1, \ldots, N_{s},
$$

where $\mathbf{L}$ is a $M \times K$ complex matrix that mixes the $K \leq M$ latent factor vectors $\mathbf{c}_{j}$. This leads to a low-rank acoustical CSM, averaged over the snapshots :

$$
\mathbf{S}_{\mathbf{a a}}=\mathbf{L S}_{\mathbf{c c}} \mathbf{L}^{H} .
$$

2. The TBL noise, which has a known correlation structure given by a Corcos-like model [8], that can also be expressed using latent variables:

$$
\mathbf{n}_{j}=\mathbf{P} v_{j}, \quad j=1, \ldots, N_{s},
$$

where $\mathbf{P}=\lceil\mathbf{p}\rfloor$ is a diagonal matrix containing the complex spectrum amplitudes $\mathbf{p} \in \mathbb{C}^{M}$. The variance of the latent variables $v_{j} \in \mathbb{C}^{M}$ is supposed to have a Corcos-like correlation shape. The noise cross-spectra between the $\mathrm{k}^{\text {th }}$ and $\mathrm{l}^{\text {th }}$ microphones with coordinates $\left(x_{k}, y_{k}\right)$ and $\left(x_{l}, y_{l}\right)$ are :

$$
\mathbb{E}\left\{n_{k} n_{l}^{*}\right\}=p_{k} p_{l}^{*} \mathrm{e}^{-\frac{2 \pi f}{U c}\left(\frac{\left|x_{k}-x_{l}\right|}{\alpha_{x}}+\frac{\left|y_{k}-y_{l}\right|}{\alpha_{y}}-l\left(x_{k}-x_{l}\right)\right)}, \quad k, l=1, \ldots, M,
$$

where $\alpha_{x}$ and $\alpha_{y}$ are the longitudinal and transverse coherence-loss and $U_{c}$ is the vortex convection velocity. This model is not totally in accordance with the 
Corcos' one since these parameters are allowed to change with frequencies as well as the power of the autospectra.

3. All the other random contributions, not carried by the previous terms, which are supposed to be uncorrelated over the microphones (e.g. ambient or electronic noise), tend to have a diagonal matrix :

$$
\mathbf{S}_{\epsilon}=\left\lceil\epsilon^{2}\right\rfloor .
$$

The proposed denoising is finally an inference problem that aims at fitting the measured CSM with the sum of these contributions :

$$
\begin{array}{cc}
\mathbf{S}_{\mathbf{p p}} & \approx \mathbf{S}_{\mathbf{a a}}+\mathbf{S}_{\mathbf{n n}}+\mathbf{S}_{\epsilon} \\
= & \mathbf{L} \mathbf{S}_{\mathbf{c c}} \mathbf{L}^{H}+\mathbf{P} \mathbf{S}_{v v} \mathbf{P}^{H}+\left\lceil\epsilon^{2}\right\rfloor .
\end{array}
$$

A Bayesian approach is adopted in order to estimate all the unknowns in Eq. (12) that best fit the data, by maximizing the posterior density function (PDF) :

$$
\mathbf{L}^{\star}, \mathbf{S}_{\mathbf{c c}}{ }^{\star}, \mathbf{p}^{\star}, \mathbf{S}_{v V}{ }^{\star}, \epsilon^{2 \star}=\operatorname{argmax}\left[\mathbf{L}, \mathbf{S}_{\mathbf{c c}}, \mathbf{p}, \mathbf{S}_{v v}, \epsilon^{2} \mid \mathbf{S}_{\mathbf{p p}}\right]
$$

where $[x \mid y]$ stands for the conditional PDF of $x$ given $y$. As the PDF in Eq. (13) has no closed-form, the optimization problem is solved numerically using the Gibbs sampler, a commonly used Monte Carlo Markov Chain algorithm [11]. The pseudo-code for this procedure is given in Algorithm 1. After a thousand iterations, the returned estimates are the mean of the last 500 draws of the Gibbs sampler.

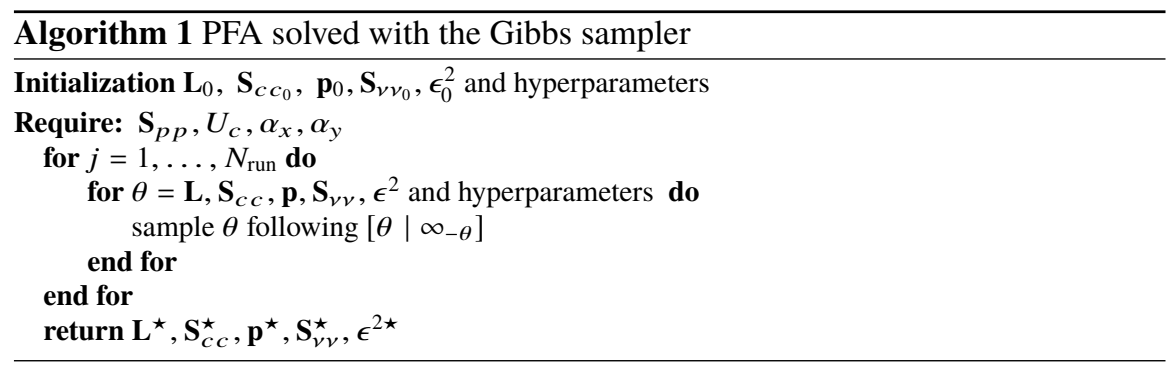

In the Bayesian approaches, all the unknowns are assigned a prior PDF, as well as the parameters (called hyperparameters) of these priors. A complex Gaussian prior is used for all the parameters, the variance of which follows itself a flat inverse gamma law. These priors and hyperpriors are gathered in Tab. 1. The parameter $v$, which describes the correlation structure of the TBL noise over the antenna is also Gaussian, with a Corcos-like variance, as given by $\mathbb{E}\left\{v v^{H}\right\}$ in Eq. (9). This variance is expressed as a function of three TBL parameters $\left(U_{c}, \alpha_{x}, \alpha_{y}\right)$. They could be inferred along with all the other unknowns of the model, but a simpler approach is to estimate them directly from the measured CSM, before applying the PFA denoising. 
This can be done by a non-linear least mean square procedure, as previously done in Ref. [3].

Previous applications of PFA for denoising have shown promising results in the medium frequency range [12,13,24], with a lack of performance in the low frequency domain, where the TBL noise is highly correlated over the microphone. This problem is here overcome by taking into account a TBL noise contribution in the inference problem. The choice of the TBL model (Corcos here) is of course important, it has to be pertinent in regard of the application case. However, the CSM associated to the TBL contribution is infered from the data, which gives a certain flexibility in order to update the model so as to make it fit to measurements. The analysis of this infered CSM is also a way to validate a posteriori the chosen model [25].

\begin{tabular}{l|l|l} 
Priors & Hyper-priors & Inputs \\
\hline$[\mathbf{L}]=\mathcal{N}_{\mathbb{C}}\left(\mathbf{0}, \mathbf{I}_{M K} / K\right)$ & & \\
{$[\mathbf{c}]=\mathcal{N}_{\mathbb{C}}\left(\mathbf{0},\left\lceil\sigma_{\mathbf{c}}{ }^{2}\right\rfloor\right)$} & {$\left[\sigma_{c}^{2}\right]=\mathcal{I} \mathcal{G}\left(\mathbf{a}_{c}, \mathbf{b}_{c}\right)$} & $\mathbf{a}_{c}, \mathbf{b}_{c}=10^{-3}$ \\
{$[\mathbf{p}]=\mathcal{N}_{\mathbb{C}}\left(\mu_{p},\left\lceil\sigma_{\mathbf{p}}{ }^{2}\right\rfloor\right)$} & {$\left[\sigma_{p}^{2}\right]=\mathcal{I G}\left(\mathbf{a}_{p}, \mathbf{b}_{p}\right)$} & $\mu_{p}=\operatorname{Trace}\left(\mathbf{S}_{\mathbf{y y}}\right) / M$ and $\mathbf{a}_{p}, \mathbf{b}_{p}=10^{-3}$ \\
{$\left[\epsilon^{2}\right]=\mathcal{I G}\left(\mathbf{a}_{\epsilon}, \mathbf{b}_{\epsilon}\right)$} & $\mathbf{a}_{\epsilon}, \mathbf{b}_{\epsilon}=10^{-3}$ \\
{$[v]=\mathcal{N}_{\mathbb{C}}\left(\mathbf{0}, \sigma_{v}{ }^{2}\left(U_{c}, \alpha_{x}, \alpha_{y}\right)\right)$} & & $U_{c}, \alpha_{x}, \alpha_{y}$
\end{tabular}

Table 1 Prior PDF assigned to each parameter and hyperparameter of the PFA model. $\mathcal{N}_{\mathbb{C}}$ stands for the multivariate complex normal distribution and $\mathcal{I G}$ is the inverse-gamma distribution. The input parameters $U_{c}, \alpha_{x}$ and $\alpha_{y}$ are given by a least mean squares procedure.

Plane wave beamforming and averaged autospectra are plotted in Fig. 10 and 11. PFA provides the same amount of denoising than the reference-based approach, but without requiring any extra noise-free measurements.

\subsection{Wavenumber filtering}

Wavenumber filtering is applied in this work only for the case of the rotating linear array, that offers the best spatial resolution. In a first section, basic principles are given for a $1 \mathrm{D}$ processing, and the extension to $2 \mathrm{D}$ is exposed in a second section.

\subsubsection{One-dimensionnal approach}

For a given angular position of the line array, the one-dimensionnal streamwise wavenumber-frequency spectrum can be directly computed by discretizing the following Fourier integral:

$$
\Phi_{p p}\left(k_{1}, \omega\right)=\frac{1}{(2 \pi)} \int S_{i j}\left(\xi_{1}, \omega\right) e^{-i k_{1} \xi_{1}} d \xi_{1}
$$



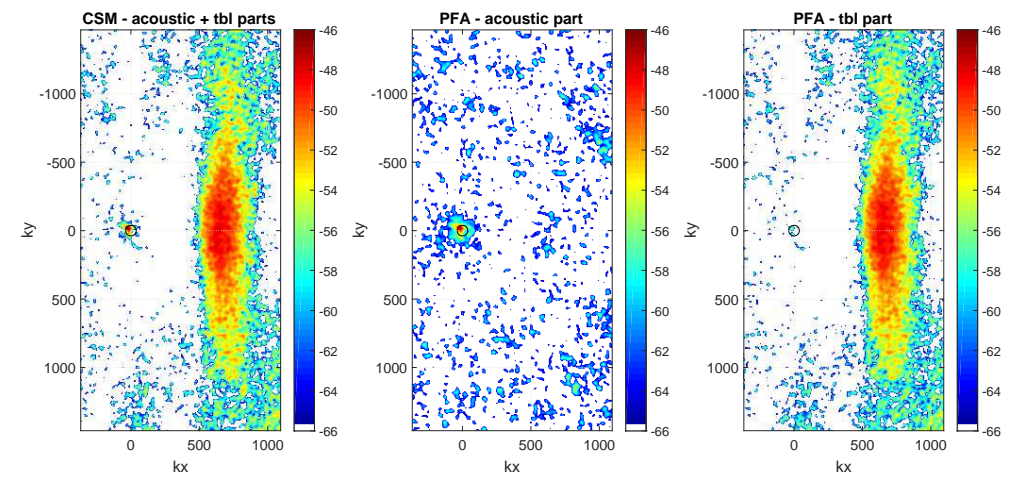

Fig. 10 Plane Wave beamforming maps obtained for the measurement configuration AT30 using the MEMS array at $2100 \mathrm{~Hz}$. Blind extration of acoustic and TBL parts using PFA. From left to right : Acoustics + TBL, Acoustics only, TBL only.

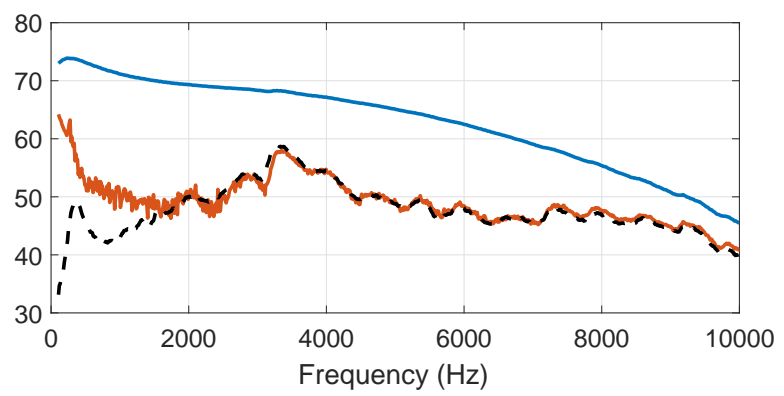

Fig. 11 MEMS array averaged Autospectra. Measurement configurations AT30 (solid blue), A (dashed black) and PFA based denoising AT30 (solid red).

where $k_{1}$ is the streamwise wavenumber. Note that the wall-pressure field is assumed to exhibit homogeneous properties over the microphone array, the cross spectrum $S_{i j}\left(\xi_{1}, \omega\right)$ is assumed to depend spatially only on the microphone spacing $\xi_{1}$. In practice, the number of elements of the CSM $(63 \times 63=3969)$ can be reduced down to approximately 500 by considering the symmetry properties of the cross-spectral matrix, and the symmetry of the line array (see Fig. 12).

The one-dimensionnal wavenumber spectrum, obtained beneath a turbulent boundary layer for increasing flow speed, with additionnal sound sources, are plotted in Fig. 13. Each plot is obtained using the same level of acoustic sound source, only the flow speed is varied from 0 to $50 \mathrm{~m} / \mathrm{s}$. At a given frequency $f_{0}$, the wavenumber spectrum is characterized by two contributions $[20,18,19,16]$ : an acoustic contribution around $k_{a}$, and an hydrodynamic contribution due to the convection 


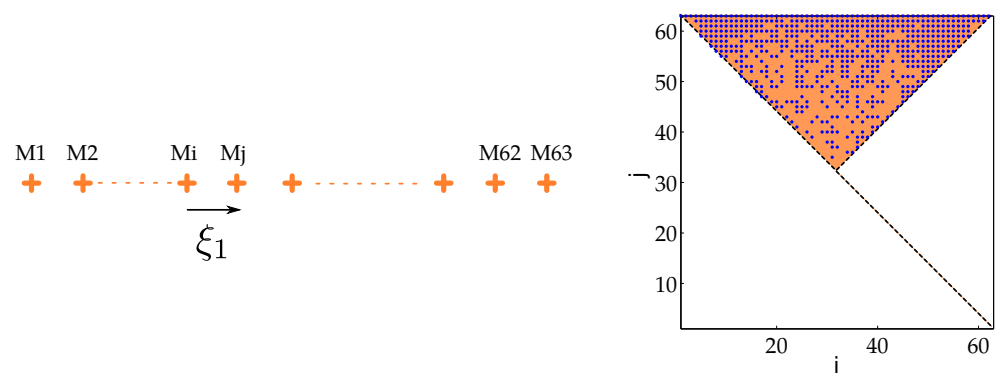

Fig. 12 One-dimensionnal case : extraction of the cross-spectral matrix with a line array of 63 microphones.

of wall-bounded turbulence, located around $k_{c}$. These two contributions are clearly visible in the spectra from Fig. 13. Furthermore, the hydrodynamic contribution can be observed by the change in its slope as the flow speed is increased.
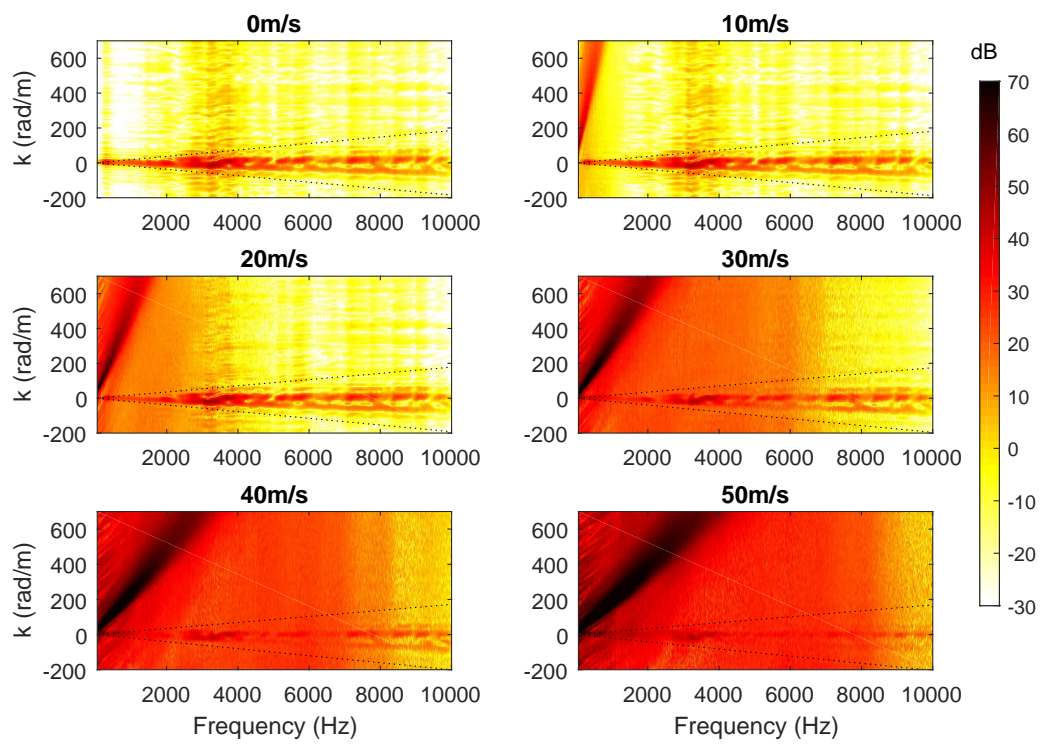

Fig. 13 One-dimensionnal wavenumber spectra beneath a turbulent boundary layer for increasing flow speed from 0 to $50 \mathrm{~m} / \mathrm{s}$, with additional sound sources.

If the frequency is higher than $500 \mathrm{~Hz}$ approximately, the wavenumber representation allows for an explicit separation between those two components. This filtering is possible by integrating all wavenumber components from the acoustic region as 


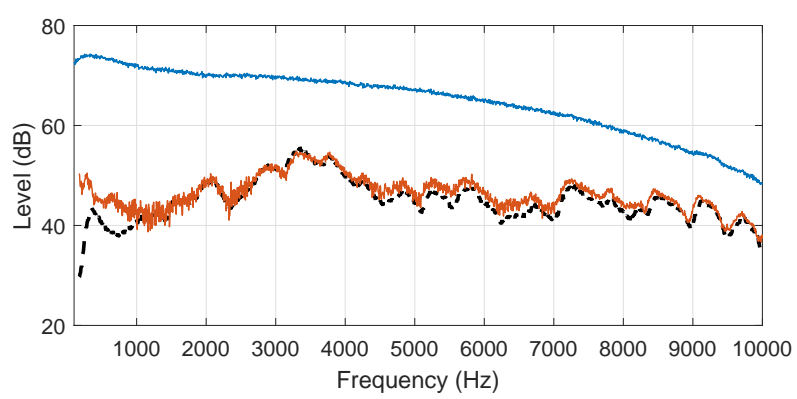

Fig. 14 Rotating linear array Autospectra (streamwise direction) for measurement configuration AT30 with (solid red) and without (solid blue) wavenumber filtering, and for configuration A (dashed black).

$$
S_{\mathrm{ii}}^{\mathrm{ac}}(\omega)=\int_{-(k 0+\Delta k / 2)}^{k 0+\Delta k / 2} \Phi_{p p}\left(k_{1}, \omega\right) \mathrm{d} k_{1} .
$$

In this example, the spatial filtering enables the direct extraction of the acoustic spectrum apart from the hydrodynamic pressure, without any assumption on the pressure field. Note that at lower frequencies (below $500 \mathrm{~Hz}$ ), the separation is not possible due to overlapping of the two contributions in the wavenumber domain. The spectral broadening, due to the limited spatial extent of the windows, is accounted for by adding the wavenumber resolution $\Delta k$ in the integration limits of Eq. (14). This approach has also been validated in the context of turbomachinery noise using a line array of MEMS microphones [22].

The resulting acoustic spectrum is plotted in red in Fig. 14 for a flow speed of $30 \mathrm{~m} / \mathrm{s}$, the acoustic spectrum obtained without flow is recalled (in dashed black). The acoustic spectrum is recovered with an agreement within $\pm 1 \mathrm{~dB}$ above $1 \mathrm{kHz}$.

\subsubsection{Two-dimensionnal approach}

The same procedure as described in section 4.3 .1 can be applied on a twodimensionnal basis using multiple angular positions of the array $[3,21]$. In that case, only cross-spectral densities of the same angular positions are computed. All spatial separations $\mathbf{x}_{i}$ can then be achieved by combining all the angular positions of the array (see Fig. 15).

The wavevector - frequency spectrum is then directly computed by discretizing the following Fourier integral,

$$
\Phi_{p p}(\mathbf{k}, \omega)=\frac{1}{(2 \pi)^{2}} \iint R_{p p}(\mathbf{r}, \omega) e^{-i \mathbf{k} \cdot \mathbf{r}} d \mathbf{r} .
$$




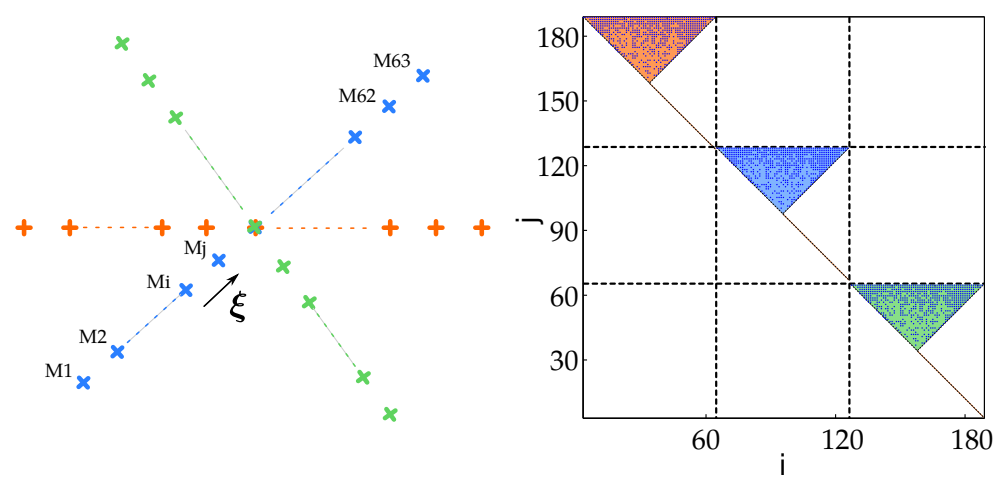

Fig. 15 Two-dimensionnal case : extraction of the cross-spectral matrix with a line array of 63 microphones and 3 angular positions, for example.

The transducer locations are denoted by $\mathbf{r}_{n m}=\left(d_{n}, \theta_{m}\right)$ in polar coordinates. For a given angle of the linear antenna, all the cross-spectra between the different microphones are available since the pressure signals are simultaneously recorded over the $n_{p}=63$ probes. The irregular radial distribution of the microphones is presented in the next section. A regular polar distribution $\theta_{m}=m \Delta \theta$ has been chosen with $\Delta \theta=\pi / m_{\theta}$ and $m_{\theta}=63$. In a straightforward approach, the wavenumber-frequency spectrum can be computed as

$$
\Phi_{p p}(\mathbf{k}, \omega)=\frac{1}{(2 \pi)^{2}} \sum_{m=0}^{m_{\theta}-1} \sum_{n=0}^{n_{r}} R_{p p}\left(\mathbf{r}_{n m}, \omega\right) e^{-i\left(k_{1} d_{n} \cos \theta_{m}+k_{2} d_{n} \sin \theta_{m}\right)} d s_{n}
$$

over a given grid $\mathbf{k}=\left(k_{1}, k_{2}\right)$. In this particular and simple case, the central microphone corresponds to $d_{0}=0$, the location of a half of the linear antenna is defined by the radial distance $d_{n}$ with $1 \leq n \leq 31$ and $n_{r}=\left(n_{p}+1\right) / 2$. The corresponding elementary area can be expressed as

$$
d s_{n}=\pi\left(l_{n+1}^{2}-l_{n}^{2}\right) \times \frac{\Delta \theta}{2 \pi}, \quad l_{n}=\frac{d_{n}+d_{n+1}}{2} .
$$

The two-dimensionnal wavenumber spectra, obtained at $30 \mathrm{~m} \cdot \mathrm{s}^{-1}$, with additional sound sources, are plotted in Fig. 16 at 1,2 and $10 \mathrm{kHz}$, for the same flow speed and the same level of acoustic sound source. These two contributions are clearly visible in the spectra from Fig. 16.

The acoustic spectrum is then obtained through the two-dimensionnal integration of the wavenumber components within the acoustic domain. The resulting spectrum is plotted in Fig. 17 and compared to the one-point spectra obtained with or without flow, using the same acoustic source. 

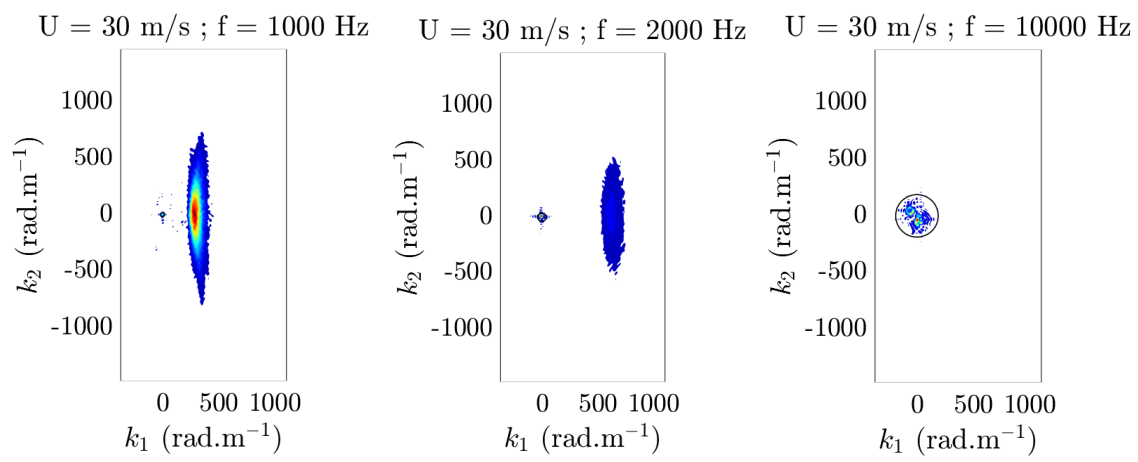

Fig. 16 Two-dimensionnal wavevector spectra beneath a turbulent boundary layer at $30 \mathrm{~m} / \mathrm{s}$, with additionnal sound sources, at $1 \mathrm{kHz}$ (left), $2 \mathrm{kHz}$ (center) and $10 \mathrm{kHz}$ (right). Solid black line : acoustic region limit defined as $|\mathbf{k}|<k_{0}$.

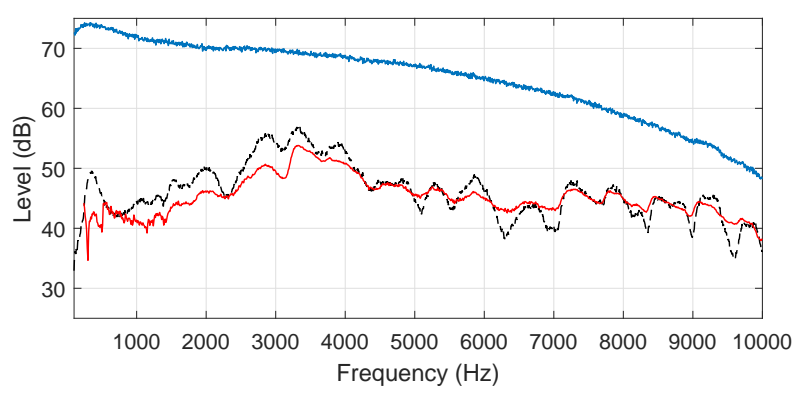

Fig. 17 Frequency spectra obtained by integrating the wavenumber components from the twodimensionnal wavenumber spectra (Fig. 16). Integration on the whole wavenumber plane (blue), in the acoustic region (red), and the spectrum without flow (black).

\subsection{Physical filtering with the accelerometer array}

The interest of using accelerometer measurements is that the thin structure on which sensors are fixed will be more efficiently excited by acoustic waves than by the turbulent structures of the flow, due to the characteristic lengths of phenomena. A pressure field will excite efficiently the plate-like structure if its wavenumber content is lower than the natural flexural wavenumber of the structure. The convective ridge of a TBL excitation, at relatively low flow speeds, goes rapidly above the flexural wavenumber when increasing the frequency, while the acoustic wavenumber stays below up to the critical frequency. In the present work, a thin aluminium plate of $1 \mathrm{~mm}$ thickness is used, its critical frequency is about $12 \mathrm{kHz}$. This means that the whole wavenumber content lying in the acoustic circle is efficiently transmitted to the plate up to $12 \mathrm{kHz}$.

The idea to use accelerometers to access to the acoustic part of an excitation field is not new, it has been investigated in several previous studies [5, 6]. In these works, an 
analytical model of the structure is used, together with a local method to identify the pressure field. This method suffers however from two drawbacks, firstly the added mass and rotation inertia of the sensors is not taken into account in the method, which can be a limitation when using a lot of sensors and a light structure, as it is the case in this work. A second drawback is that the method requires the use of a regular sampling mesh respecting the Shannon's rule (two sensors at least by natural wavelength of the structure). This would require too much sensors to cover the frequency range of interest in this work (up to $10 \mathrm{kHz}$ ). Another option is investigated in the present work: the structure, equipped with accelerometers, is characterized experimentally so as to avoid model and sampling issues. A regular excitation mesh is drawn on the frontside of the plate (the side exposed to the pressure excitation). Transfer functions between forces at excitation points and accelerations are measured using an impact hammer. The use of a spatial sampling of $15 \mathrm{~mm}$ guarantees to respect the Shannon's limit for an acoustic wave up to $10 \mathrm{kHz}$. The dimension of the plate being $30 \mathrm{~cm} \times 30 \mathrm{~cm}$, a total of 400 excitation points have to be considered, which requires a significant measurement effort. This preliminary experimental stage can be seen as a calibration of a 2D sensor constituted by the plate with its boundary conditions, and embedding the accelerometers. Note that a numerical model of the structure with sensors and cables could have been an option, with limitation related to the difficulty to correctly take into account boundary conditions and cables effects in a frequency range up to $10 \mathrm{kHz}$. The experimental option has been chosen so as to limit as much as possible bias effects that would inevitably be introduced by the use of a numerical model.

The identification of the load distribution requires to inverse a linear system with 400 unknowns (excitation dofs) and 49 equations (number of accelerometers). This inversion is underdetermined, and requires additional a priori information so as to define a unique solution. This additional information is in this work that the pressure field should be expressed in the specific basis of spherical waves generated by point sources situated at a specific distance of the array $(25 \mathrm{~cm}$ in this work, corresponding to the height of the wind tunnel in which source are located). Finally, the whole processing can be written as follows

$$
\mathbf{S}_{\mathbf{p p}}=(\Delta S)^{-2} \mathbf{G}_{\mathbf{2}}\left(\mathbf{H G}_{\mathbf{1}}\right)^{+} \mathbf{S}_{\mathbf{a a}}\left(\mathbf{H G}_{\mathbf{1}}\right)^{+H} \mathbf{G}_{\mathbf{2}}{ }^{H}
$$

where $\mathbf{G}_{\mathbf{1}}$ stands for the spherical propagator through the flow from the source plane to excitation points on the plate (analytical), $\mathbf{S}_{\mathbf{a a}}$ is the CSM of acceleration acquired during the measurement configurations, $\mathbf{H}$ is the preliminarily measured transfer matrix between excitation points and accelerometers, and $\Delta S$ the square of the discretization step of the excitation grid. $\mathbf{G}_{\mathbf{2}}$ is a second analytical acoustic transfer matrix from sources to the plate, to be defined as a function of the points where one wants to generate the pressure field. In this work, the pressure field is assessed at points corresponding to the microphone layout of the MEMS array, so as to compare results in the wavenumber domain with the same PSFs. $\mathbf{A}^{+}$denotes the regularized pseudo-inverse of $\mathbf{A}$, which is obtained using bayesian approaches (see [23] for details). 


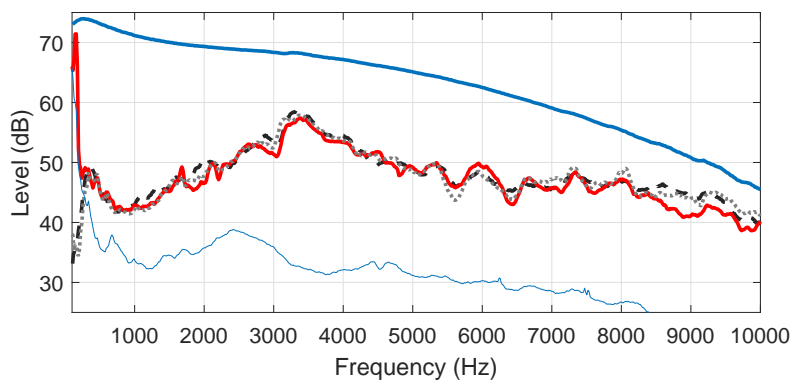

Fig. 18 Identified average spectrum from the accelerometer array at the microphone positions of the MEMS array for measurement configuration AT30 (solid red), A (dotted gray), and T30(solid thin blue). The average pressure spectrum measured with the MEMS array is recalled for comparison for the same configurations AT30 (solid blue) and A (dashed black).

The averaged pressure autospectrum identified with the accelerometer array is shown in Fig. 18, for configuration A and AT30, together with the one directly measured with the MEMS microphone array. A first observation is that the inverse procedure recovering pressures from accelerations seems to perform quite well from a quantitative point of view. The difference between the recovered pressures (configurations A or AT30) and the directly measured ones (configuration A) lays between $\pm 1 d B$ between $500 \mathrm{~Hz}$ and $5000 \mathrm{~Hz}$ (even from $100 \mathrm{~Hz}$ for the configuration without flow), and does not exceed \pm 2 to $3 \mathrm{~dB}$ up to $10 \mathrm{kHz}$. Clearly, the pressure recovered from the accelerometer array is not much sensitive to the presence of the flow: above $200 \mathrm{~Hz}$ autospectra obtained from configuration with or without the flow are very similar. The pressure autospectrum recovered for the configuration T30 (without acoustics) is also drawn in Fig. 18 so as to estimate the denoising dynamic. For the whole frequency range above $200 \mathrm{~Hz}$, the reduction as compared to the TBL pressure level measured by microphones is between 30 and $40 \mathrm{~dB}$, which illustrates well the denoising potential of this approach - at least for the studied flow speed.

Beamforming maps are displayed in Fig. 19 at a frequency of $2100 \mathrm{~Hz}$ for the pressure field identified with the accelerometer, configurations AT30, A and T30. These results can be directly compared to the ones in Fig. 4 (top figures corresponding to the MEMS array). It is verified qualitatively that the wavenumber content of the identified pressure field is not affected by the presence of the flow : the convective peak is thus efficiently filtered out by the structure for the configurations AT30 (center) and T30 (right). Note that color scales are intentionally left the same for the three maps, so as to highlight the very low level of energy in the map obtained from configuration T30 (without acoustics). 

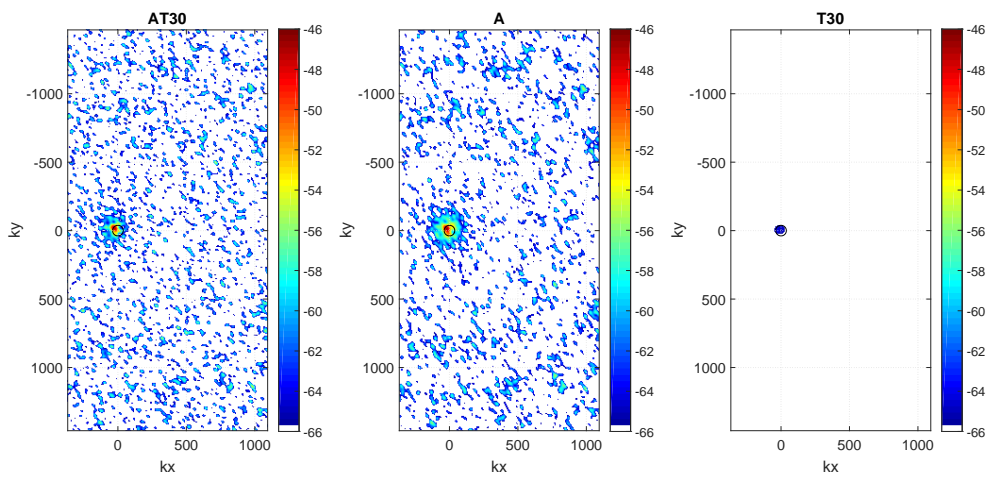

Fig. 19 Plane Wave beamforming maps for the pressure field obtained with the accelerometer array at the MEMS microphone positions, 2100Hz. From left to right : Acoustics + TBL, Acoustics only, TBL only.

\section{Conclusion}

In this chapter, different experimental measurement devices and associated post processing techniques have been presented, aiming at separating acoustic and flow contributions in a pressure field. All approaches have been applied to the same measurement configuration, so as to highlight objectively advantages and drawbacks of each. From a general point of view, the methods can be classified into two categories, depending on the hypothesis formulated on either the statistical or geometrical properties of the signals. The former can be implemented with or without reference signals, the latter results from a space domain filtering approach, either based on a high spatial sampling or using structural filtering. Of course, it could be possible to combine the different methods to eventually achieve an optimal separation, this option is left for future work. Another perspective of this work is to find indicators so as to assess objectively the quality of the separation.

\section{Acknowledgements}

This work was performed within the framework of the Labex CeLyA of Université de Lyon, operated by the French National Research Agency (ANR-10-LABX0060/ANR-16-IDEX-0005), and of the Clean Sky 2 Joint Undertaking, European Union (EU), Horizon 2020, CS2-RIA, ADAPT project, Grant agreement no 754881. 


\section{References}

1. Robert P. Dougherty. Beamforming In Acoustic Testing, pages 62-97. Springer Berlin Heidelberg, Berlin, Heidelberg, 2002.

2. Alice Dinsenmeyer, Jérôme Antoni, Quentin Leclère, and Antonio Pereira. On The Denoising Of Cross-spectral Matrices For (Aero)Acoustic Applications. In BeBec 7th Berlin Beamforming Conference, Berlin, Germany, March 2018.

3. Blandine Arguillat, Denis Ricot, Christophe Bailly, and Gilles Robert. Measured wavenumber Frequency spectrum associated with acoustic and aerodynamic wall pressure fluctuations. The Journal of the Acoustical Society of America, 128(4):1647-1655, 2010.

4. Michel Roger. Microphone Measurements in Aeroacoustic Installations. In AVT-287 STO $A V T-V K I$ Lecture Series held at the von Karman Institute, Rhode St. Genese, Belgium, May 2017.

5. D. Lecoq, C. Pézerat, J.-H. Thomas, and W.P. Bi. Extraction of the acoustic component of a turbulent flow exciting a plate by inverting the vibration problem. Journal of Sound and Vibration, 333(12):2505 - 2519, 2014.

6. Q. Leclère, E. Chéron, A. Pereira, C. Picard, and P. Souchotte. Design and experimental validation of an array of accelerometers for in-flow acoustic beamforming applications. In Proceedings of the 22nd AIAA/CEAS Aeroacoustics Conference, Lyon, France, 2016.

7. Q. Leclère, J. Antoni, E. Julliard, and A. Pintado-Peno. Consideration of estimation error in multiple coherence approaches: application to the inside/outside coherence analysis of aircraft flight tests In Proceedings of ISMA 2018, Leuven, Belgium, 2018.

8. GM Corcos. Resolution of pressure in turbulence. The Journal of the Acoustical Society of America, 35(2):192-199, 1963.

9. Robert H. Mellen. On modeling convective turbulence. Journal of the Acoustical Society of America, 88(6):2891-2893, 1990.

10. Bart A. Singer. Turbulent wall-pressure fluctuations: New model for off-axis cross-spectral density. Technical Report CR 198297, NASA, 1996.

11. Andrew Gelman, John B Carlin, Hal S Stern, David B Dunson, Aki Vehtari, and Donald B Rubin. Bayesian data analysis. Chapman and Hall/CRC, 2013.

12. Alice Dinsenmeyer, Quentin Leclere, Jérôme Antoni, and Emmanuel Julliard. Comparison of microphone array denoising techniques and application to flight test measurements. In 25th AIAA/CEAS Aeroacoustics Conference, page 2744, 2019.

13. Pieter Sijtsma, Alice Dinsenmeyer, Jérôme Antoni, and Quentin Leclere. Beamforming and other methods for denoising microphone array data. In 25th AIAA/CEAS Aeroacoustics Conference, page 2653, 2019.

14. Howard H Schloemer. Effects of pressure gradients on turbulent boundary-layer wall-pressure fluctuations. The Journal of the Acoustical Society of America, 40(5):1254-1254, 1966.

15. Theodore M Farabee and Mario J Casarella. Spectral features of wall pressure fluctuations beneath turbulent boundary layers. Physics of Fluids A: Fluid Dynamics, 3(10):2410-2420, 1991.

16. Abraham, B. M. \& Keith W. L., 1998, Direct measurements of turbulent boundary layer wall pressure wavenumber-frequency spectra, J. Fluid Eng., 120, 29-39.

17. Bendat, J.S. \& Piersol, A.G., 1986, Random data, analysis and measurement procedures, New York, John Wiley \& Sons, New York.

18. Blake, W. K., 1986, Essentials of turbulent wall-pressure fluctuations, in Mechanics of flowinduced sound and vibration, Vol. II Complex flow-structure interactions, Academic Press Inc., Orlando, Florida.

19. Bull, M. K., 1996, Wall-pressure fluctuations beneath turbulent boundary layers: some reflections on forty years of research, J. Sound Vib., 190(3), 299-315.

20. Corcos, G. M., 1964, The structure of the turbulent pressure field in boundary-layer flows, $J$. Fluid Mech., 18(3), 353-378.

21. Salze, E., Bailly, C., Marsden, O., Jondeau, E. and Juvé, D., 2014, An experimental characterization of wall pressure wavenumber-frequency spectra in the presence of pressure gradients, 20th AIAA/CEAS Aeroacoustics Conference, AIAA Paper 2014-2909. 
22. Salze, E., Jondeau, E., Pereira, A., S.L. Prigent and Bailly, C., 2019, A new MEMS microphone array for the wavenumber analysis of wall-pressure fluctuations: application to the modal investigation of a ducted low-Mach number stage, 25th AIAA/CEAS Aeroacoustics Conference, AIAA Paper 2019-2574.

23. Antonio Pereira, Jérôme Antoni, Quentin Leclere. Empirical Bayesian regularization of the inverse acoustic problem, Applied Acoustics, n. 97: p. 11-19, 2015.

24. Alice Dinsenmeyer, Jérôme Antoni, Quentin Leclère, and Antonio Pereira. A probabilistic approach for cross-spectral matrix denoising: Benchmarking with some recent methods (accepted for publication). The Journal of the Acoustical Society of America, 2020.

25. Alice Dinsenmeyer, Quentin Leclère, Jérôme Antoni, and Emmanuel Julliard. Separation of the acoustic and aerodynamic wall pressure fluctuations. In Proceedings of Forum Acusticum 2020, Lyon, France, 2020 